\title{
Modification of collagen-based sponges can induce an upshift of the early inflammatory response and a chronic inflammatory reaction led by M1 macrophages: an in vivo study
}

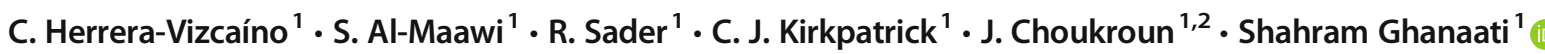

Received: 25 November 2019 / Accepted: 20 January 2020 / Published online: 17 February 2020

(C) The Author(s) 2020

\begin{abstract}
Background The present study evaluated the cellular tissue reaction of two equine-derived collagen hemostatic sponges (ECHS), which differed in thickness after pressing, over 30 days in vivo. The inflammatory response during physiological wound healing in sham-operated animals was used as control group.

Material and methods First, the E-CHS was pressed by applying constant pressure $(6.47 \pm 0.85 \mathrm{~N})$ for 2 min using a sterile stainlesssteel cylinder until the material was uniformly flattened. Consequently, the original (E-CHS), the pressed (P-E-CHS), as well as the control group (CG; sham operation) were studied independently. The 3 groups were evaluated in vivo after subcutaneous implantation in Wistar rats during 3,15, and 30 days. Histochemical and immunohistochemical methods provided observations of biomaterial degradation rate, cellular inflammatory response, and vascularization pattern. A derivative of human blood known as platelet-rich fibrin (PRF) was used as an ex vivo model to simulate the initial biomaterial-cell interaction. Segments of E-CHS and P-E-CHS were cultivated for 3 and 6 days with PRF, and the release of pro-inflammatory proteins was measured using ELISA. PRF cultivated alone was used as a control group. Results At day 3, the CG induced a statistically significant higher presence of monocytes/macrophages (CD68+), pro-inflammatory macrophages (M1; CCR7+), and pro-wound healing macrophages (M2; CD206+) compared to E-CHS and P-E-CHS. At the same time point, P-E-CHS induced a statistically significant higher presence of CD68+ cells compared to E-CHS. After 15 days, E-CHS was invaded by cells and vessels and showed a faster disintegration rate compared to P-E-CHS. On the contrary, cells and vessels were located only in the outer region of P-E-CHS and the biomaterial did not lose its structure and accordingly did not undergo disintegration. The experimental groups induced similar inflammatory reaction primarily with positive pro-inflammatory CD68+/CCR7+ macrophages and a low presence of multinucleated giant cells (MNGCs). At this time point, significantly lower CD68+/CCR7+ macrophages and no MNGCs were detected within the CG when compared to the experimental groups $(P<0.05)$. After 30 days, ECHS and P-E-CHS were fully degraded. All groups showed similar inflammatory reaction shifted to a higher presence CD206+ macrophages. A low number of CCR7+ MNGCs were still observable in the implantation bed of both experimental groups. In the ex vivo model, the cells and fibrin from PRF penetrated E-CHS. However, in the case of P-E-CHS, the cells and fibrin stayed on the surface and did not penetrate towards materials central regions. The cultivation of P-E-CHS with PRF induced a statically significant higher release of pro-inflammatory proteins compared to the CG and E-CHS after 3 days.

Conclusion Altering the original presentation of a hemostatic sponge biomaterial by pressing modified the initial biomaterial-cell interaction, delayed the early biomaterial's degradation rate, and altered the vascularization pattern. A pressed biomaterial seems to induce a higher inflammatory reaction at early time points. However, altering the biomaterial did not modify the polarization pattern of macrophages compared to physiologic wound healing. The ex vivo model using PRF was shown to be an effective model to simulate the initial biomaterial-cell interaction in vivo.
\end{abstract}

Shahram Ghanaati

Shahram.Ghanaati@kgu.de

1 Department for Oral, Cranio-Maxillofacial, and Facial Plastic Surgery, Frankfurt Orofacial Regenerative Medicine (FORM) Lab, University Hospital Frankfurt Goethe University, 60590 Frankfurt am Main, Germany

2 Pain Therapy Center, Nice, France 
Clinical relevance A pressed hemostatic sponge could be applied for guided tissue regeneration and guided bone regeneration. In that sense, within the limitations of this study, the results show that the same biomaterial may have two specific clinical indications.

Keywords Macrophages $\cdot$ Collagen-based biomaterial $\cdot$ Wound healing $\cdot$ Regeneration $\cdot$ Platelet-rich fibrin

\section{Introduction}

Research in the field of biomaterial engineering has allowed the development of biomaterials of different origins with a variety of indications. Collagen is a structural protein that awakens great interest due to its natural abundance and its presence in many biological processes [1]. As a ubiquitous and absorbable biomaterial, it has many clinical applications. For example, the literature describes the use of collagen membranes that serve as a tissue barrier for a determined amount of time and then get integrated into the implantation region [2]. The main purpose is to avoid the invasion of fast-proliferating non-osteogenic cells into bone defects. In this context, these functional collagen membranes allow osteoprogenitor cells to proliferate without the interference of soft tissue [3]. This procedure is widely applied in the field of maxillofacial surgery for bone augmentation procedures and is commonly known as guided tissue regeneration and guided bone regeneration (GTR/GBR) [3]. Additionally, collagen is especially known to be a main player in blood clot stabilization because it triggers platelet activation and consequently the release of coagulation factors [4]. Thus, the freezedrying method is a common technique used to construct hemostatic collagen-based sponges of big pores that support platelet aggregation and clot stabilization [5].

Collagen is extracted and purified from a variety of animal sources, i.e., bovine, porcine, avian, or equine, which has been described to provide the resulting biomaterials with unique physicochemical characteristics, such as pore size, pore morphology, stiffness, surface chemistry, and topography $[6,7]$. Additionally, the manufactured collagen-based biomaterials have been designed with supplementary properties to adequately fit specific indications. Ex vivo, in vivo, and clinical studies have contributed to identify the physicochemical characteristics of biomaterials as the feature determining the initial cell-biomaterial interaction, the inflammatory cellular reaction, and the vascular formation [8]. In this sense, a systematic series of studies from our group recognized biomaterials that after implantation induced a physiological cellular reaction composed of mononuclear inflammatory cells $[2,9]$. This observation derived from the subcutaneous implantation in rodents of two non-cross-linked collagen matrix of porcine ori$\operatorname{gin}[2,9]$. These histological results were correlated with those of histological samples from clinical cases, in which one of the abovementioned membranes was used to treat patients diagnosed with gingival tissue recession and for GTR of facial defects after skin cancer removal $[2,10]$. A second type of biomaterials includes those which induce the fusion of macrophages into biomaterial-related multinucleated giant cells (MNGCs). MNGCs are said to be the manifestation of a pathological cellular reaction towards the implantation of biomaterials $[11,12]$. The subcutaneous implantation of two noncross-linked, collagen-based biomaterials of porcine origin induced a pathological cellular reaction composed of MNGCs and underwent early degradation after 30 and 60 days [13]. In order to prolong the barriers stability over time, several cross-linking methods have been applied. The physical or chemical cross-linking methods include the use of ultraviolet light, hexamethylene diisocyanate, diphenylphosphoryl azide, and glutaraldehyde and aim to increase the membranes stiffness in order to slow down its reabsorption kinetics [14]. Additionally, collagen membranes have been designed as bilayers containing a spongy layer and a compact layer that avoid the complete penetration of cells. The bilayered design allows membranes to remain in the implanted region for up to 6 months $[1,15]$. However, the chemical cross-linking and the resulting physicochemical characteristics were shown to have a toxic effect on cell metabolism and induce a pathological inflammatory cellular response $[12,16]$. Natural cross-linking was recently introduced as an alternative to chemical crosslinking. This method makes use of a natural sugar molecule called ribose to reconstruct collagen fibers [17]. The studied biomaterial did not show signs of degradation after 30 days. Nevertheless, this method also induced the formation of MNGCs. Therefore, depending on the physicochemical characteristics, this type of biomaterials undergo two pathways: (a) breakdown of its native structure led by MNGCs (disintegration) and (b) induce the formation of MNGCs while retaining their space holding capacity without losing their native structure (integration) $[13,18]$.

Furthermore, macrophages are characterized by the reversible plasticity of their phenotype and functional polarization into "classically activated" macrophages (M1) or "alternatively activated macrophages" (M2). The polarization signals are provided by apoptotic cells, hormones, immune complexes, or cytokines produced by lymphocytes. It is commonly accepted that the polarization of M1 macrophages occurs in the presence of a pro-inflammatory stage during wound healing. Once the polarization into M1 takes place, they produce proinflammatory cytokines such as IFN- $\gamma$, TNF- $\alpha$, and IL-23, and express the protein chemokine receptor type 7 (CCR7) [19]. On the contrary, M2 macrophages are associated with 
tissue remodeling and wound healing. Polarization of macrophages into M2 phenotype induces the production of antiinflammatory cytokines such as IL-10, IL-1Ra, TGF- $\beta$, and the expression of the mannose receptor (MR, also known as CD206) [12, 19-22]. The reversible plasticity of macrophages and their subpopulation as part of the inflammatory body response to biomaterials continues to be a topic of research as they have been shown to mediate tissue homeostasis [19].

The categorization of the inflammatory cellular reaction to biomaterials in the aforementioned findings is the result of observational studies. Simultaneous observations of the physical and chemical characteristics make it difficult to discern between their individual effects in the inflammatory cellular reaction. It is hypothesized that modifying the original presentation of a collagen sponge, i.e., by pressing it before its implantation without further modifications, could alter the initial inflammatory cellular reaction. Therefore, the present histological study aimed to investigate the influence of modifying a non-cross-linked equine-derived collagen hemostatic sponge (E-CHS) over the inflammatory cellular reaction using the previously described ex vivo and in vivo study protocols [23]. Additionally, a pressed collagen sponge might fulfill the requirements of a collagen membrane for GBR/GTR.

\section{Material and methods}

\section{Biomaterial description}

Parasorb fleece HD® is an E-CHS indicated after tooth extraction and soft tissue injuries to achieve hemostasis. According to the manufacturer, it should be applied directly to a dry area with a light pressure for 2-3 min to achieve adhesion and stop bleeding. The sponge structure of the biomaterial allows it to absorb high quantities of fluid. This ECHS is processed through strict quality requirements to obtain a biocompatible and absorbable product of high purity.

\section{Modification of the collagen sponge biomaterial}

With the purpose of evaluating the interaction of the biomaterial's physical characteristics with its environment, the original shape of the E-CHS was altered by applying constant pressure $(6.47 \pm 0.85 \mathrm{~N})$ for $2 \mathrm{~min}$ using a sterile stainless-steel cylinder until the material was uniformly flattened (Fig. 1a, a'). The applied force was measured using a digital analytical balance. Both the E-CHS in its original form and P-E-CHS were cut into segments of $1 \mathrm{~cm}^{2}$ and assessed ex vivo and in vivo as described below. During the study, the authors refer to E-CHS and P-ECHS as two different biomaterials. Measurements of E-CHS and P-E-CHS were recorded using a digital caliper (Table 1). Images were obtained using a stereoscopic microscope (Stemi SV11; Carl Zeiss Meditec AG, Germany) (Fig. 1).

\section{Ex vivo}

The experiment was performed in triplicate, and human donors signed an informed consent after explaining to them the risks of blood withdrawal. Ethical approval for the manipulation of human samples was obtained from the ethical committee of the Goethe University of Frankfurt (IRB No. 265/17). Three healthy donors without reported blood dyscrasias or under medications participated in the study. A derivative of human blood, including a suspension of cells (platelets, leukocytes), growth factors and fibrin [24], known as liquid platelet-rich fibrin (PRF), was used as an instrument to mimic the initial biomaterial-cell interaction and to evaluate the following parameters:

a) The biomaterials' absorption coefficient

b) The fibrin's and cell's penetration within the biomaterial

c) The biomaterials' influence over the inflammatory cells in liquid PRF

As previously described, $40 \mathrm{ml}$ of blood was withdrawn according to the best practices in phlebotomy [25] into four 10-ml sterile plastic tubes (Process for PRF, Nice, France) and centrifuged using a table-top centrifuge (Duo centrifuge, Process for PRF, Nice, France; $11 \mathrm{~cm}$ radius-max, fixed angle $41.3^{\circ}$ ) following a standardized protocol known as the "lowspeed centrifugation concept" ( $600 \mathrm{rpm}, 44 \times \mathrm{g}, 8 \mathrm{~min}$ ) [26]. An average of $4 \mathrm{ml}$ of liquid PRF was obtained from each tube. Care was taken to remove only the superior liquid fraction of the tubes using a 1-ml pipette tip and used in the subsequent steps of the study. Six segments of $1 \mathrm{~cm}^{2}$ of E-CHS and P-ECHS were initially weighted, and the values were recorded as the initial weight at dry state (W0). All segments were deposited at the base of 24-well cell culture plates. Two segments were covered with distilled water and four with $1 \mathrm{ml}$ of liquid PRF. As a control measurement, $1 \mathrm{ml}$ of liquid PRF was deposited in two wells and cultivated without biomaterial. Sterile conditions were maintained during the period of cultivation. The well plates were kept for 15 min under environmental controlled conditions $\left(37{ }^{\circ} \mathrm{C}\right.$ in an atmosphere of $5 \% \mathrm{CO}_{2}$ and $95 \%$ humidity) until the liquid PRF clotted (Fig. 1E). Two segments containing distilled water and 2 with liquid PRF were removed from the cell culture plate, and the weight of the biomaterials were recorded as the weight in a wet state (W1). The liquid PRF absorption coefficient (iPAC) and the water absorption coefficient (WAC) of E-CHS and P-E-CHS were calculated using a gravimetric method according to the following equation [4]:

$\mathrm{iPAC}=\frac{\mathrm{W} 1-\mathrm{W} 0}{\mathrm{~W} 0}$

Equation 1 shows the formula to calculate the liquid PRF absorption coefficient: W0 represents the biomaterial's initial weight at dry state and W1 the weight in a wet state. 


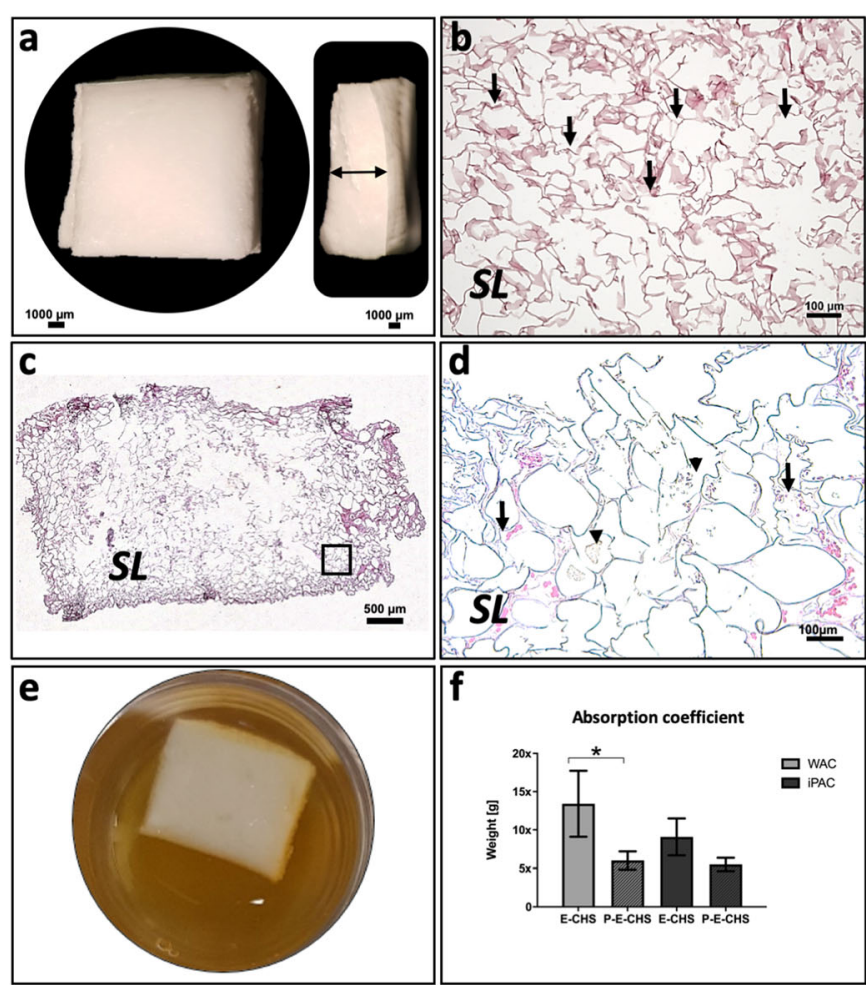

Fig. 1 Histological ex vivo evaluation of the physical characteristics of the collagen sponge in its original form (E-CHS) and pressed (P-E-CHS). (a) Overview of E-CHS's pores characteristics and the thickness of the compact $(\mathrm{CL})$ and sponge layer $(\mathrm{SL})$ (double arrow head) $(\times 20$ magnification; scale bar $=1000 \mu \mathrm{m}$ ). (b) The histological image of ECHS shows the large size of its pores, which are randomly disposed $(\times$ 200 magnification; scale bar $=100 \mu \mathrm{m})$. (c, d) Evaluation of fibrin and cell penetration into E-CHS using injectable platelet-rich fibrin (PRF). Cells and fibrin penetrated the outer third of the biomaterial (total scan; scale bar $=500 \mu \mathrm{m}$ ). At a closer magnification, cells (arrows) and fibrin (arrow heads) distributed within the large pores can be observed $(\times 200$ magnification; scale bar $=100 \mu \mathrm{m})$. (a') P-E-CHS shows a compressed structure with pores distributed horizontally $(\times 20$ magnification; scale bar $=1000 \mu \mathrm{m})$. (b) On the surface, a compressed layer (CL) is observed with a SL in the interior $(\times 200$ magnification; scale bar $=$ $100 \mu \mathrm{m}) .(\mathbf{c}$ ', d') Fibrin and cells remain on top of P-E-CHS (arrows).

Subsequently, the segments were fixed with $4 \%$ buffered formalin (Roti-Histofix 4\% acid-free pH 7, Carl-Roth, Germany) for histological evaluation. To the remaining biomaterials and liquid PRF, $1 \mathrm{ml}$ of cell culture medium (Dulbecco's modified Eagle's medium) (Biochrom GmbH, Berlin, Germany) with 1\% penicillin/streptomycin was added to the well plates for cell culture. The supernatant was collected on the 3rd and 6th days and stored at $-80{ }^{\circ} \mathrm{C}$. The concentration of interleukin 8 (IL-8) and tumor necrosis factor-alpha (TNF- $\alpha$ ) were measured in the collected supernatants using a quantitative sandwich DuoSet ELISA kit (R\&D Systems, Minneapolis, USA) following the manufacturer's instruction. The absorbance assay was conducted using a microplate reader (Infinite M200, Tecan, Grödig, Austria) set to a wavelength of $450 \mathrm{~nm}$ with a reference reading at $570 \mathrm{~nm}$. The calculated concentrations of E-CHS and P-E-CHS were relatively quantified with respect to liquid PRF without biomaterial.

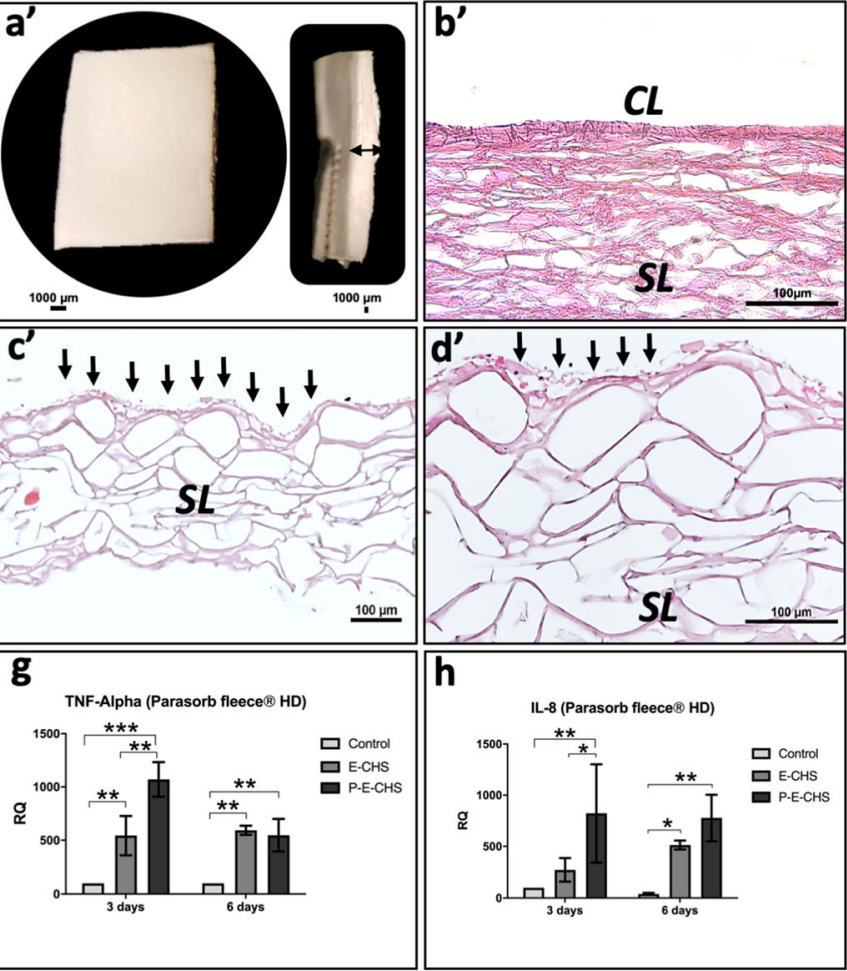

After cultivation with injectable PRF the structure of P-E-CHS changes resulting in pores of larger size; however, cells and fibrin remain on top of the biomaterial $(\times 40$ and $\times 200$ magnifications; scale bar $=100 \mu \mathrm{m})$. The histological evaluation was performed using hematoxylin and eosin staining. (e) Representative image of the biomaterials cultivated with injectable PRF in cell culture well plates. (f) Water and injectable PRF absorption coefficient (WAC, iPAC) of E-CHS and P-E-CHS. (g, h) Inflammatory protein profile of liquid injectable PRF cultivated with ECHS and P-E-CHS compared to PRF without biomaterial as a control measurement. The release of TNF-alpha and IL- 8 in the experimental groups has statistically significant increased compared to control. Results were relative quantified (RQ) to the first measurement at day 3. Results are presented as mean \pm standard deviation. Differences were considered statistically significant if the $P$ values were $<0.05\left(^{*}\right)$ and highly significant if the $P$ values were $<0.01(* *),<0.001(* * *)$, and $<0.0001(* * * *)$.

\section{In vivo}

The animal experiment was designed and conducted according to the ARRIVE guidelines and the EU Directive 2010/63/ EU for animal experiments [27]. Ethical approval was obtained from the regulating authorities of Darmstadt, Germany and the ethical committee of the University of Frankfurt (Approval No. FK/1023). Animals were housed in groups under hygiene standards in the animal facility (ZTE) belonging to the Department of Medicine of the University of Frankfurt. Thirty Wistar rats (Rattus norvegicus) were purchased from Charles River (Sulzfeld, Germany) aged 6 to 8 weeks, weighting approx. 200 g. Prior to surgery, the animals were kept 7 days for acclimatization under control conditions (Temp $20^{\circ} \mathrm{C}$, light/dark cycles of $12 \mathrm{~h}$ and humidity of 40 to $70 \%$ ). They were fed with rodent pallets and water ad 
libitum. The animals were divided into two groups of 12 animals; group 1 was implanted with E-CHS and group 2 with P-E-CHS. Additionally, 4 animals per time point (12 in total) were included as a control group (Sham operation). Anesthesia was induced through an intraperitoneal injection mixture of ketamine/xylazine $(100 \mathrm{mg} / \mathrm{kg} / 5 \mathrm{mg} / \mathrm{kg})$. The animals were placed in prone position and anesthetic depth was verified by the absence of the toe reflex. Under sterile conditions, a subcutaneous pocket was prepared by performing a 2$\mathrm{cm}$ skin incision in the infrascapular area and dissection of the subcutaneous tissue. The biomaterial was cut in segments of $1 \mathrm{~cm}^{2}$ and placed above the muscle's fascia. The tissue was sutured using a 4-0 polypropylene (Prolene Ethicon, NJ, USA). Follow-up was carried out according to a standard score sheet, and analgesia was managed with tramadol (1-3 $\mathrm{mg} / \mathrm{kg} / \mathrm{day})$ administered orally through the drinking water for 1 day. At each time point, 3, 15, and 30 days post-operatively, 4 animals per group and 4 shamoperated animals were euthanized with an anesthetic overdose. The biomaterials with the surrounding tissue were explanted and preserved in $4 \%$ buffered formalin (RotiHistofix 4\% acid-free $\mathrm{pH} 7$, Carl-Roth, Germany) during $24 \mathrm{~h}$ for further histological evaluation.

\section{Histological/immunohistological staining and qualitative evaluation}

As previously described [9], the samples were divided into $2 \mathrm{~mm}$ segments and processed using an automatic tissue processor (Leica TP1020). Paraffin blocks were sectioned in 3$4 \mu \mathrm{m}$ slides using a microtome (Leica RM2255, Wetzlar, Germany) and stained with hematoxylin and eosin (H\&E) as a first general screening step. The slides obtained from the paraffin blocks representing the most optimal cross-section of the biomaterials, at each time point of explantation and group, were selected for further evaluation. From each paraffin block, 7 consecutive slides were histologically stained as follows: 1st H\&E, 2nd Azan, 3rd Mazon Goldner; the remaining 4 slides were immunohistochemically (IHC) stained using an autostainer (Lab Vision ${ }^{\mathrm{TM}}$ Autostainer 360, Thermo Scientific), and the antigen retrieval was performed using the heat-induced epitope retrieval (HIER) method using a water bath (VWR $®$, Germany) at $95{ }^{\circ} \mathrm{C}$ for $20 \mathrm{~min}$ (Table 2). The 4th slide was stained IHC with CD 68 antibody (pan marker for the monocyte/macrophages linage) and the 5th with $\alpha$ Smooth Muscle Actin ( $\alpha$-SMA; vessel identification). The 6th slide was IHC stained with CD206 (M2 macrophages) and the 7th with CCR7 (M1 macrophages). Additionally, one slide from the samples was selected randomly and added as a negative control without the application of the primary antibody. The resulting slides were qualitatively evaluated using a light microscope (Nikon Eclipse 80i, Tokyo, Japan). Representative histological images were captured with a
Table 1 Physical measurements of the collagen sponge (E-CHS) in its original presentation and after pressing (P-E-CHS)

\begin{tabular}{llll}
\hline Biomaterial & Height $(\mathrm{mm})$ & Volume $\left(\mathrm{mm}^{3}\right)$ & Surface area $\left(\mathrm{mm}^{2}\right)$ \\
\hline E-CHS & 5 & 500 & 400 \\
P-E-CHS & 0.5 & 50 & 220 \\
\hline
\end{tabular}

Nikon DS-Fi1 digital camera and a Nikon Digital sight unit DS-U3 (Nikon, Tokyo, Japan) and controlled running the Nikon's NIS-Elements imaging software.

\section{Quantitative histomorphometric evaluation}

Through an automatic procedure and using the automatically motorized stage of a Nikon ECLIPSE 80i histological microscope, a total of 150-200 images were taken at $\times 20$ magnification from each slide. The large image settings from NISElements software (Nikon, Tokyo, Japan) was used to form a large image or "total scan," allowing the total visualization of the sample in one single image and the histomorphometric analyses.

\section{Biomaterial degradation}

The degradation rate of E-CHS and P-E-CHS in vivo was measured by performing measurements of the implantation bed after 3,15, and 30 days. Using the NIS-Elements software (Nikon, Tokyo, Japan), fifteen cross-sectional measurements distributed longitudinally through the biomaterial were recorded and the results were expressed in square millimeters and as the mean (SD) of the measurements from 4 samples (4 different animals) at each time point. The results from days 15 and 30 were compared to day 3 and statistically analyzed.

\section{Category of the cellular inflammatory reaction}

Using the manual "annotations and measurements" tool from NIS-Elements software, the presence of MNCs (CD68+), MNGCs (CD68+/CCR7+), M2 macrophages (CD206+), and $\mathrm{M} 1$ macrophages $(\mathrm{CCR} 7+)$ were quantified. From each individual slide at 3 and 15 days (4 per time point and group), the total number of counted cells was divided by the total area of the implantation bed as a relative measurement to the cellular reaction of E-CHS and P-E-CHS. At 30 days of implantation, due to biomaterial degradation and wound healing, a mean area obtained from the measurements of all slides was used as normalization to define the inflammatory cellular reaction. The results were expressed as CD68+ MNCs $/ \mathrm{mm}^{2}$ (M1/M2) and MNGCs $/ \mathrm{mm}^{2}$ with statistical intragroup and intergroup comparison between time points. 


\section{Vascularization pattern}

The vascular structures were identified by the formation of a clear lumen, cell deposition, and the positive immunostaining with $\alpha$-SMA. Using the "area annotation" tool of the NISElements software, the vascular structures in the implantation bed and within the biomaterial were manually delineated. The results were expressed firstly as "density vascularization," calculated as the total number of vascular structures divided by the total area of the biomaterial and its implantation bed. Secondly, the "percentage vascularization" was calculated by dividing the total lumen area by the total biomaterial's area. The results were expressed as vessel per square meter and percent respectively with statistical intragroup and intergroup comparison between time points.

\section{Statistics}

Primary end points were the characterization of the initial biomaterial-cell interaction and the inflammatory cellular reaction in terms of the induction of MNGCs and the vascularization pattern. The secondary outcomes were absorption coefficient (iPAC); biomaterial degradation; and the signaling of molecule expressions CD68, CCR7 (M1), and CD206 (M2). Sample size was calculated $(n=4)$ according to previous studies [17, 28, 29]. Statistical analyses were carried out using GraphPad Prism 7.0 software (GraphPad Software Inc., La Jolla, CA, USA). All results of the ex vivo assay and in vivo experiments were analyzed using two-way analysis of variance (ANOVA) with a Tukey multiple comparisons test ( $\alpha=$ $0.05,95 \% \mathrm{CI}$ of diff.) of all pairs. The results are presented as the mean and standard deviation (SD) and depicted in graphs. Intragroup (Control group •) (E-CHS •) (P-E-CHS •)/intergroup $(*)$ differences were considered statistically significant with a significance level of $P<0.05$.

\section{Results}

\section{Ex vivo}

After applying constant pressure to E-CHS for $2 \mathrm{~min}$, it did not recover its shape at dry stage (Table 1). Three layers are observable in both biomaterials: a superior and inferior thin and compact layer (CL) and a middle sponge layer (SL) (Fig. 1a, a'). Histologically, the pore's geometry of E-CHS is of irregular shape and of bigger size compared to P-E-CHS, which pores are horizontally disposed (Fig. 1b, b'). In the case of E-CHS, the fibrin entered the biomaterial and formed a network inside the pores. Additionally, the cells are penetrating the outer third of the biomaterial's body, but without reaching the center region (Fig. 1c, d). The observations in P-E-CHS are the opposite; the biomaterial's structure prevented the fibrin and the cells to enter the biomaterial's body as they are only observed on the surface (Fig. 1c', d'). Furthermore, the results exhibited E-CHS as a hemostatic sponge with a mean WAC of 13-fold and an iPAC of 8-fold its original weight. The results showed a reduction of the mean WAC and iPAC capacity of P-E-CHS to 6- and 5.6-fold, respectively. The WAC from E-CHS showed statistically significant results compared to P-E-CHS $(P<0.05 *)$ (Fig. 1e, f).

The measurements of the pro-inflammatory proteins TNF- $\alpha$ and IL- 8 were barely measurable in the CG (PRF alone) at 3 days and 6 days. In both experimental groups (PRF + E-CHS and PRF + P-E-CHS), a change in the protein profile was detected. There was a significant increase of TNFalpha after 3 days in the PRF + P-E-CHS group compared to the CG $(P<0.001 * * *)$ and to the PRF + E-CHS group $(P<0.01 * *)$. The measurements of the PRF + E-CHS group were also significantly higher than the $\mathrm{CG}(P<0.01 * *)$ at this time point. Furthermore, a higher release was measured at 6 days in both experimental groups compared to the $\mathrm{CG}$ $(P<0.05 *)$, while no differences were observed when comparing E-CHS and P-E-CHS. P-E-CHS's release of IL-8 was higher with statistically significant results compared to the $\mathrm{CG}$ $(P<0.01 * *)$ and to PRF + E-CHS at day $3(P<0.05 *)$. After 6 days, both E-CHS and P-E-CHS showed a higher release of IL-8 with statistically significance compared to the CG $(P<0.05 *$ and $P<0.01 * *$ respectively). No differences were measured between P-E-CHS and E-CHS (Fig. 1g, h).

\section{In vivo}

All animals survived the surgery and wounds healed uneventfully during the study without adverse reaction. No signs of loss of body weight or exacerbating inflammatory signs were recorded at any of the study time points.

\section{Qualitative histological analyses}

After 3 days, the highest number of macrophages (CD68+) was observed in the control group (CG; sham-operated) surrounding the subcutaneous pocket. The presence of CCR7+ cells (M1 macrophages) was slightly higher than CD206+ cells (M2 macrophages). The observed vascular structures were of native origin. Thereafter, the presence of the inflammatory cells progressively reduced at day 15 and day 30 . Nevertheless, at 30 days, the observed number of CD206+ cells was higher than the number of CCR7+ cells. No MNGCs were present during the observation period. An increase in vascular structures was detected at day 15 with a posterior reduction after 30 days. In the experimental groups, after 3 days of implantation, both biomaterials were found in the implantation bed with no signs of material degradation. A change in the porous size in the outer third of E-CHS was observed, as it appears to be slightly pressed. The remaining two most interior thirds of E-CHS 


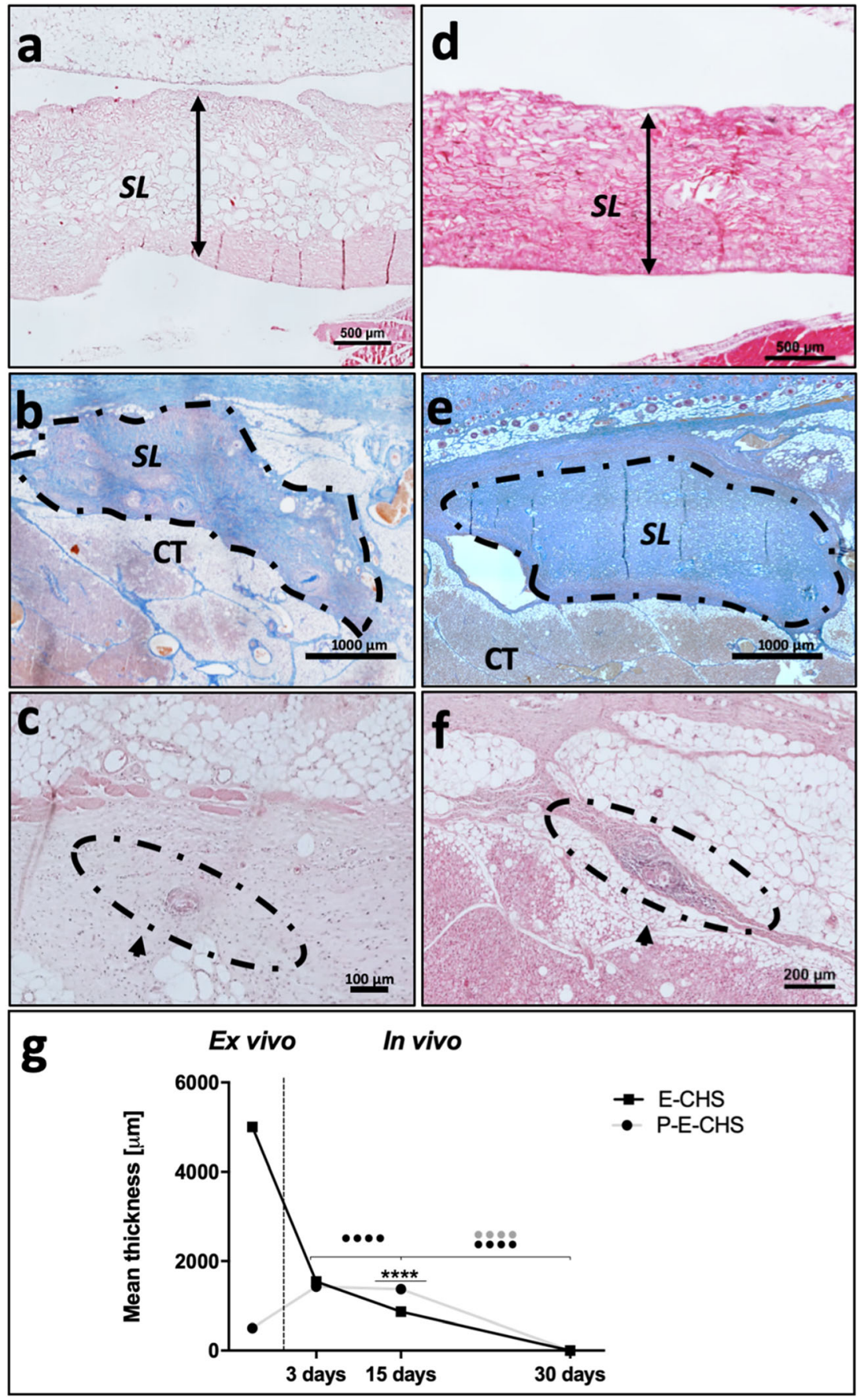

Fig. 2 In vivo biomaterial degradation after subcutaneous implantation in Wistar rats (Rattus norvegicus). The collagen sponge (a-c) in its original form (E-CHS) and (d-f) pressed (P-E-CHS). (a) After 3 days postimplantation, E-CHS outer third is compressed and the sponge layer (SL) preserved the pore structure (hematoxylin and eosin staining $(\mathrm{H} \& \mathrm{E}) ; \times 40$ magnification; scale bar $=500 \mu \mathrm{m})$. (d) P-E-CHS remains compressed and the pores are observed disposed horizontally $(\mathrm{H} \& \mathrm{E} ; \times 40$ magnification; scale bar $=500 \mu \mathrm{m})$. (a, d) The double arrow head indicates the measurements of a cross-section of the biomaterials. (b) After 15 days post-implantation, cells have invaded E-CHS completely and it is showing signs of degradation (Azan staining; "total scan"; scale bar $=1000 \mu \mathrm{m}$ ). (e) On the contrary, cells remain on the surface and outer third of P-E-CHS with no signs of biomaterial degradation (Azan staining; "total scan"; scale bar $=1000 \mu \mathrm{m})$. (b, e) The dash lines represent the perimeter of the biomaterials. (c-f) After 30 days postimplantation, remaining fragments of E-CHS and P-E-CHS are observed occupied by inflammatory cells $(\mathrm{H} \& \mathrm{E} ; \times 200$ magnification; scale bar $=100 \mu \mathrm{m})$. The dash lines represent the analyzed implantation bed. (g) Histomorphometric analyses of biomaterial degradation. Intragroup $(\bullet, \bullet)$ and intergroup $(*)$ differences were considered statistically significant if the $P$ values were $<0.05 \bullet, \bullet / *$ and highly significant if the $P$ values were $<0.01 \bullet \cdot, \bullet / * *,<0.001 \bullet \bullet, \cdots * * * *$, and $<0.0001 \cdots . ., \cdots * * * * * *$ 

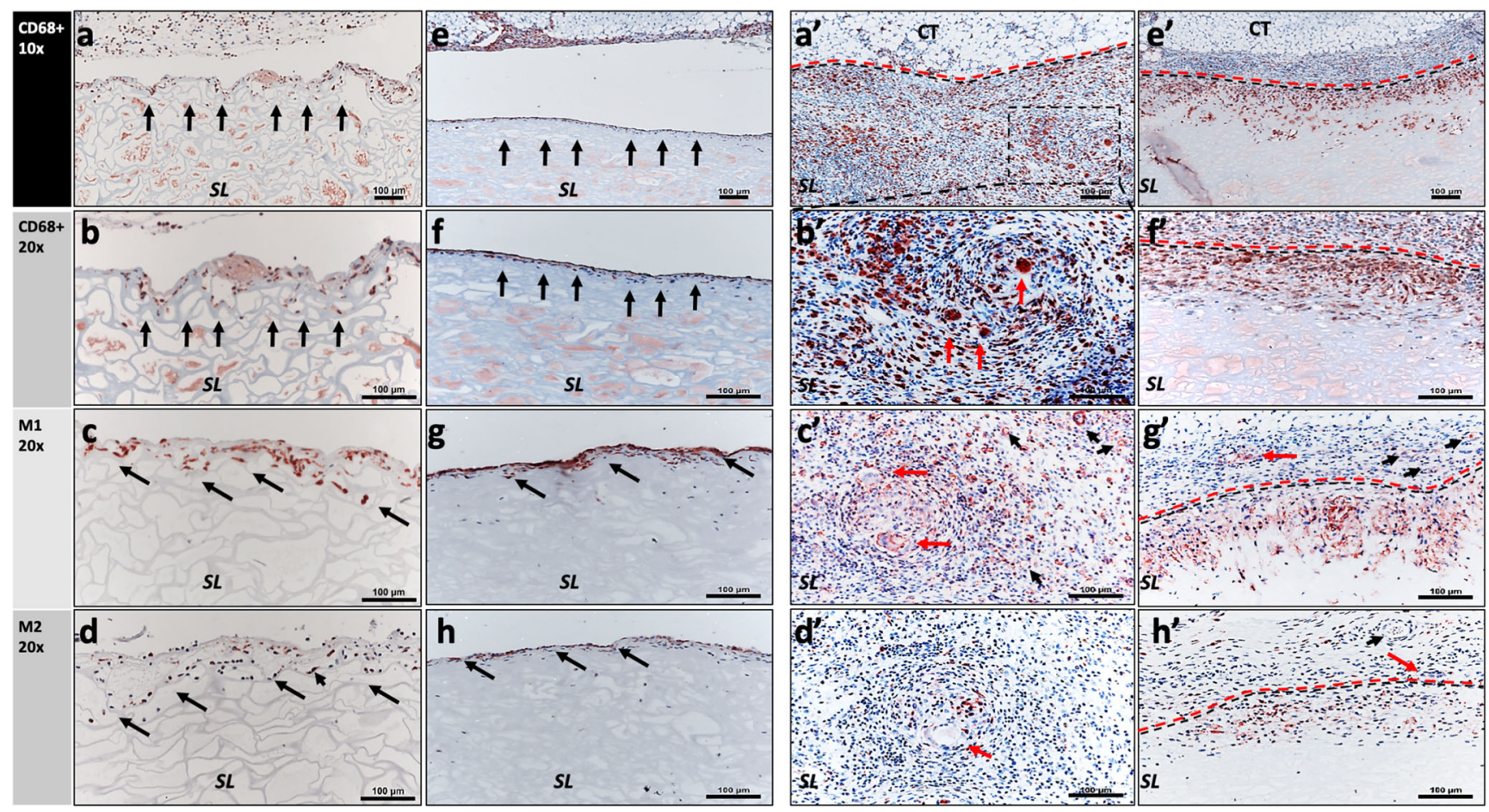

Fig. 3 Immunohistochemical evaluation of the inflammatory cellular reaction and the polarization of macrophages into M1 and M2 phenotypes. The collagen sponge (a-d) in its original form (E-CHS) and (e-h) pressed (P-E-CHS) at $3(\mathbf{a}-\mathbf{h})$ and $15\left(\mathbf{a}^{\prime}-\mathbf{h}^{\prime}\right)$ days after subcutaneous implantation in Wistar rats (Rattus norvegicus). (a, e) CD68-positive cells (brown) in E-CHS and P-E-CHS showing the early inflammatory cellular reaction and the initial biomaterial-cell interaction (CD68 immunohistochemical (IHC) staining; $\times 100$ magnification; scale bar $=100 \mu \mathrm{m}$ ). (b) Cells of the macrophage lineage penetrated the sponge layer (SL) of E-CHS (arrows) (CD68 IHC staining; $\times 200$ magnification; scale bar $=100 \mu \mathrm{m}$ ). (f) In the case of P-E-CHS, cells of the macrophage lineage are observed on the surface of the biomaterial (CD68 IHC staining; $\times 200$ magnification; scale bar $=100 \mu \mathrm{m}$ ). (c) Macrophages of M1 phenotype (brown) are mainly observed penetrating E-CHS (arrows) in a higher number than P-E-CHS, (g) where the macrophages are observed on the surface (CCR7 IHC staining; $\times 200$ magnification; scale bar $=100 \mu \mathrm{m})$. (d-h) Fewer macrophages of M2 phenotype (brown) are observed in both biomaterials (CD206 IHC staining; $\times 200$ magnification; scale bar $=100 \mu \mathrm{m}$ ). (a') After 15 days post-implantation, a higher inflammatory cellular reaction was observed in E-CHS, which is completely penetrated by mononuclear cells and a smaller number of

maintained its structure and the space of the implantation area (Fig. 2a). P-E-CHS preserved the appearance of a pressed structure at this time point. Its pores are of smaller size forming rectangular shapes and disposed horizontally (Fig. 2d). At this time point, the biomaterial-cell interaction of E-CHS and P-ECHS was different. Inflammatory cells surrounded the surface of E-CHS, and the cells penetrated the outermost third of ECHS's body. In the case of P-E-CHS, inflammatory cells were surrounding the surface and cells accumulated on the surface of the biomaterial. In both biomaterials, the inflammatory reaction consists of MNCs, which are mostly CD68+/CCR7+ (Fig. 3c, $\mathrm{d}, \mathrm{g}, \mathrm{h})$. At this time point, MNGCs were not observed in the implantation bed. multinucleated giant cells (MNGCs). (b') IHC staining showed CD68+ cells (brown) of the immune lineage, i.e., macrophages and MNGCs (red arrow) $($ CD68 IHC staining; $\times 100$ and $\times 200$ magnifications; scale bar $=$ $100 \mu \mathrm{m})$. The red dash line delineates the biomaterial's body (black) and the surrounding implantation area (red). (e', f') The inflammatory cellular reaction increased also in the implantation bed of P-E-CHS compared to day 3 , but the positively stained cells did not enter the biomaterial's center. In this scenario, the inflammatory cellular reaction was dominated by macrophages (CD68 IHC staining; $\times 100$ and $\times 200$ magnifications; scale bar $=100 \mu \mathrm{m}$ ). (c') Mainly macrophages of M1 phenotype and MNGCs expressing CCR7 marker (brown) are observed in the center of E-CHS and penetrating blood vessels (black arrow) $(\times 200$ magnification; scale bar $=100 \mu \mathrm{m}$ ). (g') P-E-CHS induced a inflammatory cellular reaction with a higher number of M1 macrophages, a reduced number of MNGCs (red arrow) and vessels (black arrow) surrounding the biomaterial (CCR7 IHC staining; $\times 200$ magnification; scale bar $=100 \mu \mathrm{m})$. (d', h') The CD206 IHC staining showed positively stained M2 macrophages (brown), negatively stained MNGCs (red arrow), and vessels in the implantation area (black arrow) $(\times 200$ magnification; scale bar $=100 \mu \mathrm{m})$

After 15 days of implantation, E-CHS and P-E-CHS were found in the implantation bed but P-E-CHS was showing higher signs of degradation. Two different cell-biomaterial interactions were again observed at this time point. E-CHS was well integrated into the implantation bed and its structure and pores are hardly identified as the biomaterial's body was fully invaded by cells and granulation tissue (Fig. 2b). The presence of vascular structures was identified in the implantation bed and inside the biomaterial's center (Fig. 6a). P-E-CHS integrated into the implantation bed, but the cells were mainly surrounding the biomaterial and invaded only the outer-third of the biomaterial's body (Fig. 2e). Vascular structures were mainly observed surrounding the biomaterial and the implantation bed 


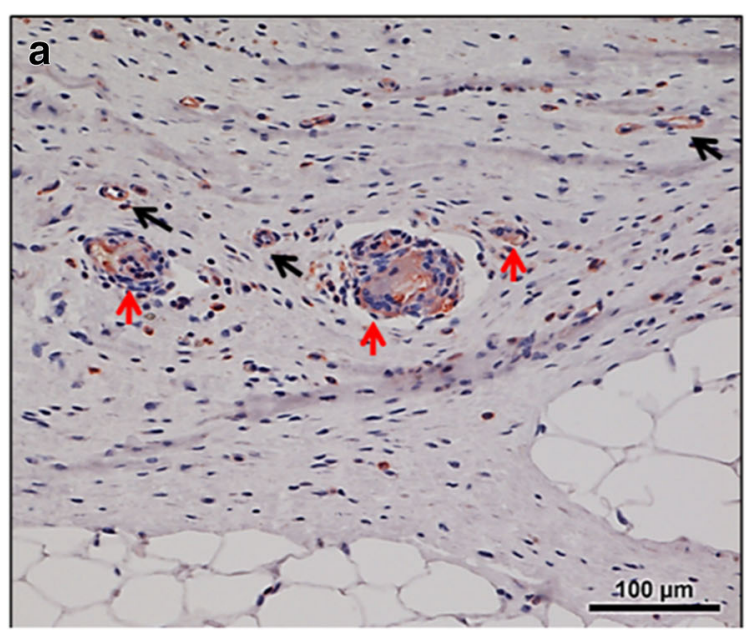

Fig. 4 Biomaterial fragmentation and inflammatory cellular reaction at 30 days after subcutaneous implantation. (a) The fragments of the collagen sponge in its original form (E-CHS) and (b) pressed (P-E-CHS)

(Fig. 6b). In both groups, a higher number of inflammatory cells were observed compared to day 3 consisting of a higher number of CD68+/CCR7+. A reduced number of MNGCs were observed at this time point (Fig. 3a'-h').

By day 30 of implantation, E-CHS and P-E-CHS fully degraded and inflammatory cells were observed surrounding the remaining fibrils of the biomaterials. A low number of residual macrophages, CD68+/CCR7+ MNGCs, and micro-vessels were observed within the implantation bed (Figs. 2c, f and 4).

\section{Quantitative histomorphometric evaluation}

\section{Biomaterial thickness}

The histomorphometric measurements of the implantation bed taken on day 3 showed that E-CHS and P-E-CHS had comparable mean thickness without statistical differences at this time point. After 15 days of implantation, the intragroup comparison showed that E-CHS had a thickness reduction of 0.7 -fold, which was statistically significant $(P<0.0001$ •.••) compared to day 3. The structure of P-E-CHS did not disintegrate as fast as E-CHS and after 15 days of implantation had a thickness reduction of 0.3 -fold. The results showed statistically significant thickness reduction of E-CHS compared to P-E-CHS at this time point $(P<0.0001 * * * *)$.After 30 days post-implantation, only small fragments of E-CHS and P-E-CHS were found in the implantation area $(P<0.0001 \cdots \cdots / . \cdots * \bullet)$ (Fig. 2g).

\section{The inflammatory pattern of macrophages}

Three days after implantation, the highest number of CD68+, CCR7+, and CD206+ MNCs were found in the implantation bed of the CG with statistically significant difference compared to E-CHS $(P<0.001 * * * ; P<0.0001 * * * * ; P<0.001 * * *$,

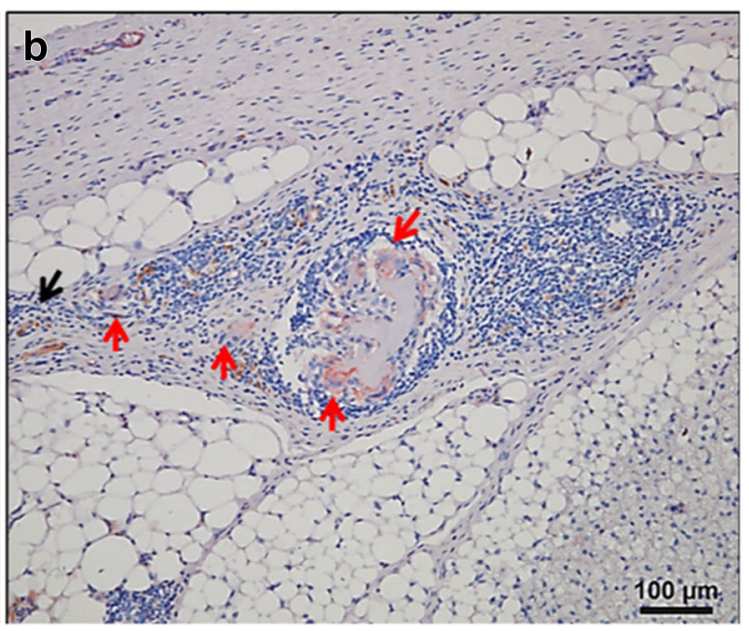

are surrounded by remaining macrophages, multinucleated giant cells (red arrows), and vessels (black arrows) (CCR7 immunohistochemical (IHC) staining; $\times 200$ and $\times 100$ magnifications; scale bar $=100 \mu \mathrm{m}$ )

respectively) and P-E-CHS $(P<0.05 * ; \quad P<0.0001 * * * *$; $P<0.001 * * *$, respectively) (Fig. 5a-f). The experimental groups did not show intergroup statistically significant differences at this time point. After 15 days, the number of CD68+ MNCs in the CG considerable reduced compared to day 3 $(P<0.01 \cdot-)$. The experimental groups showed the opposite cellular reaction with an increase of CD68+ MNCs in the implantation bed. The intragroup comparison showed that the number of CD68+ MNCs observed in E-CHS at this time point was statistically significantly higher compared to day 3 $(P<0.01 \bullet \bullet)$. Additionally, the CD68+ cells in the E-CHS group at this time point were statistically significantly higher compared to the $\mathrm{CG}(P<0.05 *)$. The number of pro- and antiinflammatory macrophages (CCR7+; M1/CD206+; M2) in the CG followed the same tendency as the CD68+ MNCs. After 15 days of implantation, there was a reduction in the number of CCR7+ $(P<0.001 \cdot-\cdot)$ and CD206+ $(P<0.05 \cdot)$ MNCs in the $\mathrm{CG}$, which was statistically significant lower compared to day 3. The pro- and anti-inflammatory macrophages observed in the experimental groups showed at this time point a different polarization pattern. A significant increase of CCR7+ MNCs was observed in both biomaterials. However, only the number of CCR7+ MNCs observed in P-E-CHS at day 15 was statistically significant compared to day $3(P<0.05 \bullet)$. The intergroup evaluation showed that the higher number of CCR7+ MNCs measured in E-CHS and P-E-CHS at this time point was statistically significant compared to the $\mathrm{CG}(P<0.01 * *$; $P<0.05 *)$. Interestingly, the number of CD206+ MNCs in the implantation bed of both biomaterials showed comparable values to those measured at day 3 . At day 30 , a significant reduction of CD68+ and CCR7+ MNCs was observed in the control and the experimental groups. Nevertheless, the number of CD68+ MNCs measured in P-E-CHS continues to be higher compared to the $\mathrm{CG}$ with statistical significance $(P<0.05 *)$. 

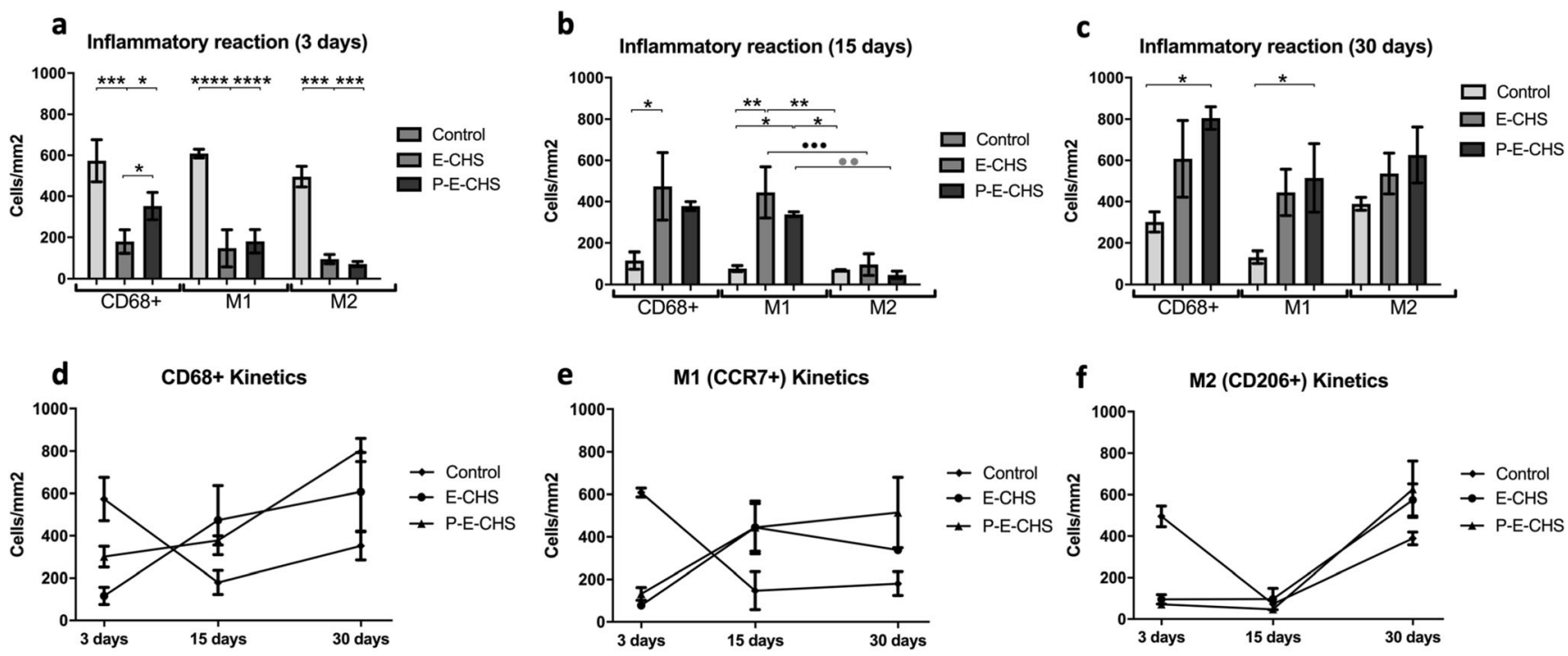

Fig. 5 Histomorphometric analyses of the inflammatory cellular reaction. (a, d-f) At 3 days post-implantation, a higher number of CD68+, M1, and $\mathrm{M} 2$ cells $/ \mathrm{mm}^{2}$ were counted in the control group (Sham operation) compared to the experimental groups (E-CHS and P-E-CHS). At the same time point, P-E-CHS induced a statistically significant higher presence of CD68+ macrophages compared to E-CHS. (b, d-f) After 15 days, a higher inflammatory reaction $\left(\right.$ cells $/ \mathrm{mm}^{2}$ ) in E-CHS and PE-CHS was measured, being statistically significant compared to control.

(c, d-f) After 30 days post-implantation, the same trend of the inflammatory cellular reaction was observed in the control and experimental groups with a higher presence of M2 macrophages. Intragroup $(\bullet, \bullet)$ and intergroup $(*)$ differences were considered statistically significant if the $P$ values were $<0.05 \bullet, \bullet / *$ and highly significant if the $P$ values were, $<0.01 \bullet \cdot, \cdot * * *,<0.001 \cdot \cdots, \cdot \cdots * * * *$, and $<0.0001 \cdots \cdots, \cdots \cdots * * * *$

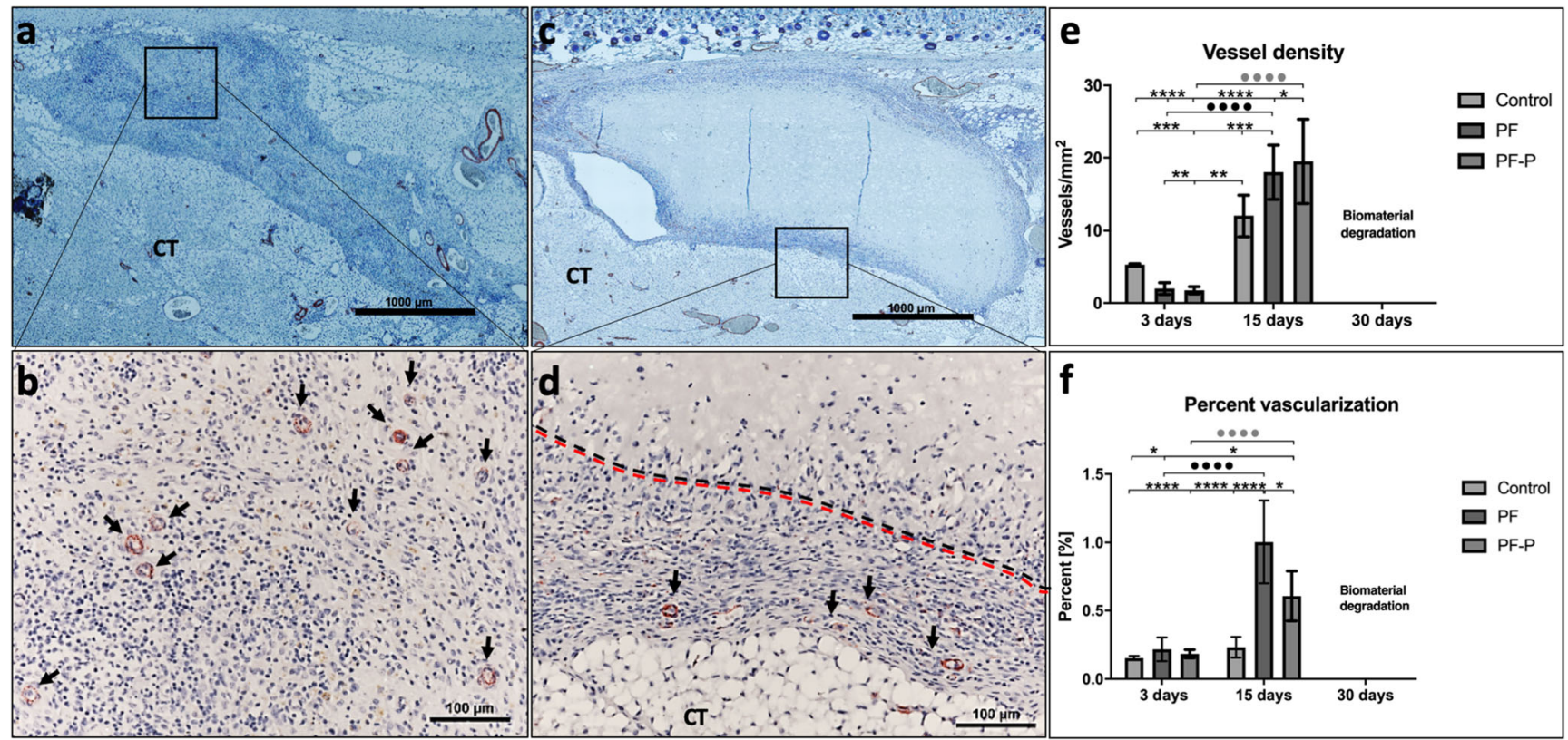

Fig. 6 Biomaterial vascularization pattern. (a) The collagen sponge in its original form (E-CHS) was invaded completely by cells after 15 days of subcutaneous implantation. Vessels (brown) formed in the implantation bed and inside the biomaterial's body (arrows) ( $\alpha$-SMA IHC staining; "total scan"; scale bar $=1000 \mu \mathrm{m}$. (b) $\alpha$-SMA IHC staining; $\times 200$ magnification; scale bar $=100 \mu \mathrm{m}$ ). (c) At 15 days of implantation, cells are observed surrounding the pressed biomaterial (P-E-CHS). (d) The red dash line delineates the biomaterial's body (black) and the surrounding implantation area (red). Vessels were formed only in the implantation bed (arrows) ( $\alpha$-SMA IHC staining; "total scan"; scale bar $=1000 \mu \mathrm{m}$. (d) $\alpha$-SMA IHC staining; $\times 200$ magnification; scale bar $=100 \mu \mathrm{m}$ ). (e-f) After 3 and 15 days the density (vessels $/ \mathrm{mm}^{2}$ ) and percent [\%] of vessel were histomorphometrically analyzed. After 30 days, the biomaterial degraded and remaining fragments of E-CHS and P-E-CHS were occupied by inflammatory cells. Intragroup $(\bullet, \bullet)$ and intergroup (*) differences were considered statistically significant if the $P$ values were $<0.05 \bullet, \bullet * *$ and highly significant if the $P$ values were $<$

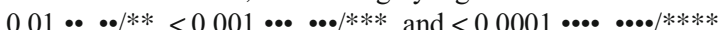


At this time point, remarkably, there was a shift of the polarization pattern of all groups towards a higher number of CD206+ MNCs compared to the MNCs expressing the proinflammatory marker CCR7, thus without statistically significant results (Fig. 5c, f).

\section{Vascularization pattern}

The density and percentage area of vascularization were evaluated within the implantation bed and inside the biomaterial's body (Fig. 6e, f). The results at day 3 in the CG, E-CHS, and PE-CHS showed comparable results with no statistical differences at this time point. At day 15, the measurements increased in all groups considerably. However, the results in the CG were no statistically significant compared to day 3 . The E-CHS and P-E-CHS groups, on the other hand, showed a significantly higher vessel density and percentage area of vascularization compared to day $3(P<0.0001 \cdots \cdots, \cdots$, , respectively). At this time point, the intergroup comparison showed statistically significant higher vessel density in the E-CHS group compared to the $\mathrm{CG}$ at day $15(P<0.0001 * * * *)$. Additionally, the results showed statistically significant higher percent of vascularization in the experimental groups compared to the CG (E-CHS: $P<0.0001 * * * *$; P-E-CHS: $P<0.05 *$ ). On day 30 , only a reduced number of vessels were observed at the implantation bed and surrounding small fragments of the biomaterial.

\section{Correlation of the inflammatory cellular reaction ex vivo and in vivo to the biomaterials}

The results ex vivo and in vivo showed the same pattern of inflammatory reaction. At day 3 , the measurements ex vivo of TNF- $\alpha$ and IL- 8 of the P-E-CHS group induced a higher release of TNF-alpha and IL- 8 compared to E-CHS $(P<0.01 * *)$. In vivo, after 3 days, similar results were observed in the P-ECHS group with a higher inflammatory CD68+ cells compared to the E-CHS group $(P<0.05 *)$. Moreover, after 6 days of cultivation ex vivo, both experimental groups showed higher release of TNF- $\alpha$ and IL-8 compared to the CG and no differences between them. The same reaction was observed after 15 days in vivo, as both experimental groups induced a higher inflammatory reaction compared to the $\mathrm{CG}$, while there were no differences between them (Table 3).

\section{Discussion}

The aim of the present investigation was to evaluate the effect of the physical characteristics of a biomaterial in the inflammatory cellular reaction and have a closer look into the biomaterial-cell interaction. For this purpose, an E-CHS was selected because it allows a change of its original presentation without chemical modifications. Additionally, a recently introduced method was put in practice to characterize the biomaterial-cell interaction ex vivo and in vitro. Liquid platelet-rich fibrin was used as a model to reproduce the initial contact between inflammatory cells and biomaterials. The rationale behind the use of PRF lies in its high content of inflammatory cells and the depleted number of erythrocytes. Liquid PRF is a blood-derived cell concentrate of autologous use in the clinical practice to improve wound healing, reduce pain and for the supplementation of acellular biomaterials with living cells prior to implantation - a procedure known as "biologization" [30-32]. Recent studies from our research group are providing histological insights as to how biologization occurs $[17,33]$. Furthermore, the production of PRF matrices following the systematic approach of the LSCC showed in vitro and in vivo induction of angiogenesis [34-36]. Moreover, the biomaterials were evaluated in vivo following an established method of subcutaneous implantation in rodents. This animal model has shown its relevance as a mean to understand the inflammatory cellular reaction to biomaterials. The histological results of systematically conducted studies in Wistar rats have shown its similarities to histological results from clinical studies [2].

It was observed ex vivo that pressing E-CHS turned the biomaterial into a compact structure. It was also observed that once the biomaterials came into contact with distilled water and liquid PRF, the pores expanded and the biomaterials absorbed a high quantity of fluid. This is of relevance, as the capacity to absorb fluid has been linked to higher hemostatic performance and increased tissue regeneration $[37,38]$. In this sense, we introduced for the first time the iPAC measurement as a tool to determine the functionality of biomaterials to

Table 2 Antibodies used to categorize the cellular inflammatory reaction and vascularization pattern

\begin{tabular}{|c|c|c|c|c|}
\hline Antibody & Dilution & $\begin{array}{l}\text { Incubating } \\
\text { time (min) }\end{array}$ & Secondary antibody & Detection method \\
\hline Anti-Human CD68 antibody (MCA341GA, Bio-Rad, USA) & $1: 400$ & 30 & Goat anti-mouse IgG-H & AEC \\
\hline Anti-Actin, $\alpha$-Smooth Muscle antibody (A5228, Merck KGaA, Germany) & $1: 20.000$ & 120 & Goat anti-mouse IgG-H & AEC \\
\hline Anti-CCR7 antibody [Y59] (ab32527, Abcam, UK) & $1: 1000$ & 30 & Goat anti-rabbit IgG-B & AEC \\
\hline Anti-CD206 antibody (ab64693, Abcam, UK) & $1: 1000$ & 30 & Goat anti-rabbit IgG-B & $\mathrm{AEC}$ \\
\hline
\end{tabular}


Table 3 Correlations of the inflammatory cellular reaction ex vivo and in vivo. The control groups were taken as reference measurements

\begin{tabular}{|c|c|c|c|}
\hline In vivo & & Ex vivo & \\
\hline Days & Control & Days & PRF-Control \\
\hline 3 & $\begin{array}{l}\text { High inflammatory reaction } \\
\uparrow \mathrm{CD} 68+ \\
\uparrow \mathrm{CCR} 7+ \\
\uparrow \mathrm{CD} 206+\end{array}$ & 3 & $\begin{array}{l}\text { Low inflammatory reaction } \\
\downarrow \text { TNF-alpha } \\
\downarrow \text { IL-8 }\end{array}$ \\
\hline 15 & $\begin{array}{l}\text { Low inflammatory reaction } \\
\downarrow \text { CD68+ } \\
\downarrow \text { CCR7+ } \\
\downarrow \text { CD206+ }\end{array}$ & 6 & $\begin{array}{l}\text { Low inflammatory reaction } \\
\downarrow \text { TNF-alpha } \\
\downarrow \text { IL-8 }\end{array}$ \\
\hline 30 & $\begin{array}{l}\text { Low inflammatory reaction } \\
\downarrow \text { CD68+ } \\
\downarrow \text { CCR7+ } \\
\uparrow \text { CD206+ }\end{array}$ & & \\
\hline In vivo & & PRF & \\
\hline Days & E-CHS & Days & PRF + E-CHS \\
\hline 3 & $\begin{array}{l}\text { Lower inflammatory response } \\
\downarrow \text { CD68+ } \\
\downarrow \text { CCR7+ } \\
\downarrow \text { CD206+ }\end{array}$ & 3 & $\begin{array}{l}\text { Higher inflammatory reaction } \\
\uparrow \text { TNF-alpha } \\
\uparrow \text { IL-8 }\end{array}$ \\
\hline 15 & $\begin{array}{l}\text { Higher inflammatory reaction } \\
\uparrow \text { CD68+ } \\
\uparrow \text { CCR7+ } \\
\downarrow \text { CD206+ }\end{array}$ & 6 & $\begin{array}{l}\text { Higher inflammatory reaction } \\
\uparrow \mathrm{TNF} \text {-alpha } \\
\uparrow \mathrm{IL}-8\end{array}$ \\
\hline 30 & $\begin{array}{l}\text { Higher inflammatory reaction } \\
\uparrow \text { CD68+ } \\
\downarrow \text { CCR7+ } \\
\uparrow \text { CD206+ }\end{array}$ & & \\
\hline In vivo & & PRF & \\
\hline Days & P-E-CHS & Days & PRF + P-E-CHS \\
\hline 3 & $\begin{array}{l}\text { Lower inflammatory reaction } \\
\downarrow \text { CD68+ } \\
\downarrow \text { CCR7+ } \\
\downarrow \text { CD206+ }\end{array}$ & 3 & $\begin{array}{l}\text { Higher inflammatory reaction } \\
\uparrow \mathrm{TNF} \text {-alpha } \\
\uparrow \mathrm{IL}-8\end{array}$ \\
\hline 15 & $\begin{array}{l}\text { Higher inflammatory reaction } \\
\uparrow \text { CD68+ } \\
\uparrow \text { CCR7+ } \\
\downarrow \text { CD206+ }\end{array}$ & 6 & $\begin{array}{l}\text { Higher inflammatory reaction } \\
\uparrow \text { TNF-alpha } \\
\uparrow \text { IL-8 }\end{array}$ \\
\hline 30 & $\begin{array}{l}\text { Higher inflammatory reaction } \\
\uparrow \mathrm{CD} 68+ \\
\downarrow \mathrm{CCR} 7+ \\
\uparrow \mathrm{CD} 206+\end{array}$ & & \\
\hline
\end{tabular}

transport liquid PRF. Furthermore, it was measured in vivo an increase of the size of P-E-CHS histomorphometrically, which is consequent with the observations ex vivo. Interestingly, although P-E-CHS remain permeable to the surrounding fluid with an increase of its pore's size ex vivo and in vivo, at 15 days post-implantation, it remained cell-occlusive, as the cells did not penetrate the biomaterial's center (Fig. 2e, g). A similar biomaterial-cell interaction was observed in a previous study from our group. A cross-linked collagen membrane of bovine origin was implanted subcutaneously in Wistar rats and after 10 days of subcutaneous implantation, it increased its size 0.5 -folds. After 30 days of observation, the collagen membrane retained its barrier function [33]. Studies at the nanoscale have shown that during the first contact of a biomaterial with blood, proteins are absorbed at the biomaterial's surface. The absorbed proteins create an interface that regulates the functionality of the immune cells $[39,40]$. The results from this study support the assumption that the biomaterial- 
cell interaction and the inflammatory cellular reaction are highly determined by their initial contact. Notably, the shift in size after biomaterial implantation did not modify the subsequent inflammatory cellular reaction.

Further studies have described that variations in the pore size, pore geometry, stiffness, and surface topography also modulate the inflammatory environment [41]. In the present study, the ex vivo results indicate that the combination of PRF and biomaterials significantly enhanced the release of TNF-a and IL-8 compared to PRF alone. Additionally, changing ECHS to a pressed biomaterial induced the highest stimulus of the inflammatory cells within liquid PRF (Fig. 1g, h). The evaluated pro-inflammatory proteins ex vivo, IL- 8 and TNF- $\alpha$, have been reported to be potent inflammatory chemokines that induce neutrophil migration [42]. In this sense, the higher release of TNF- $\alpha$ and IL- 8 induced by the biomaterials ex vivo might explain the higher number of CD68+ MNCs observed in the implantation bed of the biomaterials in vivo compared to the CG after 15 days. Besides the induction of neutrophil migration, IL- 8 has been described to be a potent pro-angiogenic chemokine that upregulates the vascular endothelial growth factor (VEGF) mRNA in endothelial cells [43]. The results provide a rationale behind the improvement of wound healing in clinical studies when liquid PRF is applied in combination with biomaterials [31]. In summary, after modifying E-CHS by pressing it, the two biomaterials showed different biomaterial-cell interaction and the pressed biomaterial stimulated a higher release of liquid PRF's secretome ex vivo.

In vivo, the inflammatory cellular reaction was primarily integrated by macrophages during all time points with a small number of MNGCs. Furthermore, cells penetrated completely E-CHS and only partially P-E-CHS after 15 days. This category of inflammatory cellular reaction led by macrophages, prone to biomaterial's macrophage degradation accompanied by enzymatic lysis, has been previously reported by our research group [10]. Using the subcutaneous implantation model, the inflammatory cellular reaction to a bilayer, non-crosslinked matrix, manufactured from collagen I/III and harvested from porcine's skin and peritoneum, was investigated over 60 days. By the end of the study, the histological observations depicted a membrane undergoing degradation performed by macrophages in the absent of MNGCs [2]. A different inflammatory cellular reaction was observed in a further collagen membrane with analogous characteristics (i.e., bilayered, non-cross-linked, manufactured with collagen type I/III and harvested from pericardium). After 60 days, the compact layer of the biomaterial was not penetrated by cells and the surrounding inflammatory cellular reaction induced by the spongy layer showed a high number of MNGCs [18]. The presence of MNGCs led to a total disintegration of the spongy layer and loss of the initial structure. The physical characteristics of a biomaterial that allow cells to penetrate the biomaterials' body seem to diminish the fusion of macrophages into MNGCs independently of the chemical properties and the degradation pattern. The fusion of macrophages was previously described as a frustrated attempt of phagocytosis [44]. In this sense, there may be other factors stimulating the fusion of macrophages into MNGCS than mainly frustrated phagocytosis. It has been shown that biomaterials of reduced porosity and increased stiffness are less susceptible to degradation and provide a higher surface area for cells to attach and spread, leaving the cell's surface for a longer time exposed to body-fluid shear forces [45]. Previous studies, for example, have shown that the effect of the body-fluid shear forces over endothelial cells stimulate a higher release of VEGF [45]. Thus, the body-fluid shear forces might be stimulating the fusion of macrophages into MNGCs and at the same time inducing angiogenesis by stimulating the release of VEGF from the surrounding endothelial cells (EC) $[23,45,46]$. In the case of cross-linked collagen biomaterials, this assumption is supported by previous findings from our group where the presence of MNGCs is generally surrounding the broken down segments during biomaterial disintegration. The same can be said of MNGCs surrounding granules of bone-substitute biomaterials independent of their morphology and tissue of origin [17, 47]. Biomaterial's segmentation during the disintegration process may translate into a higher surface area and thus a higher number of cells exposed to body fluid shear forces. This would explain the steep increase in the number of MNGCs observed during the disintegration process of biomaterials.

In the present study, both biomaterials induced a reduced number of MNGCs at 15 and 30 days of implantation. In both time points, they were expressing a pro-inflammatory (CCR7+) marker rather than an anti-inflammatory (CD206+) marker. Contrary to the macrophages, the remaining MNGCs in the implantation area continue to express the pro-inflammatory marker CCR7 after 30 days, confirming their involvement in chronic inflammation [23]. These results are in accordance with a recently published histological study in humans that investigated biopsies of patients undergoing sinus floor elevation with bone substitute biomaterials. Similar to the present study, it was concluded that independently of the physicochemical characteristics of the biomaterials, after 6 months, MNGCs expressed the pro-inflammatory marker CCR7 [11].

In order to further understand how the physical characteristics of both biomaterials influenced the inflammatory cellular reaction, the polarization pattern of macrophages was identified using immunohistochemistry. It was observed that macrophage's polarization was dominated by M1 macrophages (pro-inflammatory) during the course of the study. The presence of M2 macrophages did not greatly vary after 3 and 15 days post-implantation and remained in a lower number compared to M1 macrophages during the early stages of healing. After 30 days, the implantation area seems to be 
achieving homeostasis and entering a reparative stage as a similar shift towards an anti-inflammatory pattern was observed in the control and the experimental groups, characterized by a transition towards a higher presence of M2 macrophages. It is not possible to precise exactly when this shift took place, as no observations were taken between days 15 and 30 . Nevertheless, the implantation of biomaterials may have induced a chronic inflammatory cellular reaction with a higher presence of M1 macrophages that extended until day 15 of implantation and regressed to a similar wound healing process as the CG after 30 days. Although M2 macrophages are said to have an anti-inflammatory effect due to the release of antiinflammatory growth factors, the results of our study indicate that the reduced number of M2 macrophages at early stages of wound healing might be playing a regulatory role that avoids the exacerbation of inflammation. A recent study has shown that biologic materials, i.e., extracellular matrix scaffolds, induce higher expression of the pro-inflammatory CD206+ marker on macrophages at an early time point of wound healing [41]. It may be favorable for the regeneration process to stimulate M2 macrophages at earlier time points. However, if this translates into clinical observations of accelerated wound healing requires further research. Based on our results, a pressed biomaterial did not change the physiologic polarization pattern of macrophages. Further studies with a detailed screening of the cytokine profile after biomaterial implantation, together with gene knockdown animal models, may help further characterize macrophage polarization.

Numerous characteristics of collagen biomaterials are associated with the animal source, the harvesting compartment, and the manufacturing process. For example, matrix porosity and pore morphology were shown to be animal sourcedependent [48]. A study comparing collagen extracted from bovine, porcine, and avian source concluded that the bovine and avian collagen produced collagen sponges with the smallest and largest pores, respectively $[6,48]$. An additional study demonstrated the variability of the inflammatory cellular reaction implanting subcutaneously in mice 2 collagenbased biomaterials extracted from the same species but harvested from different compartments (Pericardium vs. Dermis) [18]. Based on our findings, it is feasible to assume that the same applies for the E-CHS, providing a different chemical composition as well as physical properties compared to other animal sources. The question that rises is whether the results are reproducible using hemostatic non-cross-linked collagen sponges of different chemical compositions or origins.

Taken together, the data in this study add to the growing body of evidence that exhibits the presence of a cell sensingmechanism being triggered by the physical characteristics of biomaterials during the initial biomaterial-cell interaction [49, 50]. Consequently, modulation of the inflammatory pattern by the physicochemical characteristics of biomaterials and biomaterials with a shape-shifting capacity post-implantation could have future clinical indications. A limitation of the study may be the absence of characterization of the mechanical properties and surface chemistry of the collagen sponge after pressing. The mechanical characterization, although important, is out of the scope of this study, as the authors sought to conduct a histological analysis focused on the inflammatory cellular response. For this reason, the authors simulated the handling of the collagen sponge as it would be handled in the clinical practice.

According to our findings, it is clinically relevant that pressing the collagen sponge during its application reduces the size of the sponge's porosity, delays its early degradation rate, diminishes the absorbance coefficient, and avoids vessel formation in its interior. Applying a pressed biomaterial to control hemorrhage in bone defects could function during the healing process as a space holder biomaterial, however, undergoing disintegration before bone formation and leading to a collapse of the treated bone defect, i.e., in cases of bone healing of the alveolar socket after tooth extraction [49]. On the other hand, a pressed biomaterial could be indicated for GBR/GTR. In that sense, within the limitations of this study, the results show that the same biomaterial may have two specific clinical indications. The present findings call for translational studies to further understand the implications of biomaterial handling and the effect of their modification in the clinical practice.

\section{Conclusion}

The present study evaluated the influence of modifying a collagen sponge biomaterial over the inflammatory cellular reaction ex vivo and in vivo. Based on our results, a collagenderived hemostatic sponge of equine origin induced a chronic inflammation with a higher presence of pro-inflammatory macrophages and a low number of MNGCs. Pressing the hemostatic sponge before its implantation modified the initial biomaterial-cell interaction and altered the degradation rate of the biomaterials. The ex vivo study using platelet-rich fibrin showed to be a reliable model to simulate the initial biomaterial-cell interaction. Altogether, it is here shown the relevance of the preclinical characterization of the tissue reaction to biomaterials using animal models. The results bring to attention the importance of the correct manipulation of biomaterials during their application in the clinical practice. Recent practices attempt to enhance biomaterials with different additives - such as platelet concentrates - in an effort to accelerate wound healing. In this regard, altering the original presentation of a biomaterial may result in unexpected clinical outcomes that call for further histological studies. Finally, the results provide insight into the possibility of developing versatile biomaterials with multiple applications. 
Funding information Open Access funding provided by Projekt DEAL. This work was supported by the German Dental Research Association (Forschungsgemeinschaft Dental e.V. project Nr. 06/2017).

Compliance with ethical standards Ethical approval for the manipulation of human samples was obtained from the ethical committee of the Goethe University of Frankfurt (IRB No. 265/17). The animal experiment was designed and conducted according to the ARRIVE guidelines and the EU Directive 2010/63/EU for animal experiments [27]. Ethical approval was obtained from the regulating authorities of Darmstadt, Germany and the ethical committee of the University of Frankfurt (Approval No. FK/1023).

Conflict of interest The authors declare that they have no conflict of interest.

Ethical approval All applicable international, national and institutional guidelines for the care and use of animals were followed. All procedures performed in studies involving human participants were in accordance with the ethical standards of the institutional and/or national research committee and with the 1964 Helsinki declaration and its later amendments or comparable ethical standards

Informed consent Informed consent was obtained from all individuals participants included in the study.

Open Access This article is licensed under a Creative Commons Attribution 4.0 International License, which permits use, sharing, adaptation, distribution and reproduction in any medium or format, as long as you give appropriate credit to the original author(s) and the source, provide a link to the Creative Commons licence, and indicate if changes were made. The images or other third party material in this article are included in the article's Creative Commons licence, unless indicated otherwise in a credit line to the material. If material is not included in the article's Creative Commons licence and your intended use is not permitted by statutory regulation or exceeds the permitted use, you will need to obtain permission directly from the copyright holder. To view a copy of this licence, visit http://creativecommons.org/licenses/by/4.0/.

\section{References}

1. Sorushanova A, Delgado LM, Wu Z et al (2019) The collagen suprafamily: from biosynthesis to advanced biomaterial development. Adv Mater 31:1-39. https://doi.org/10.1002/adma. 201801651

2. Ghanaati S, Schlee M, Webber MJ et al (2011) Evaluation of the tissue reaction to a new bilayered collagen matrix in vivo and its translation to the clinic. Biomed Mater 6:015010. https://doi.org/10. 1088/1748-6041/6/1/015010

3. Elgali I, Omar O, Dahlin C, Thomsen P (2017) Guided bone regeneration: materials and biological mechanisms revisited. Eur J Oral Sci 125:315-337. https://doi.org/10.1111/eos.12364

4. Cheng X, Shao Z, Li C et al (2017) Isolation, characterization and evaluation of collagen from jellyfish Rhopilema esculentum Kishinouye for use in hemostatic applications. PLoS One 12:121. https://doi.org/10.1371/journal.pone.0169731

5. Schoof H, Apel J, Heschel I, Rau G (2001) Control of pore structure and size in freeze-dried collagen sponges. J Biomed Mater Res 58: 352-357. https://doi.org/10.1002/jbm.1028

6. Parenteau-Bareil R, Gauvin R, Cliche S, Gariépy C, Germain L, Berthod F (2011) Comparative study of bovine, porcine and avian collagens for the production of a tissue engineered dermis. Acta Biomater 7:3757-3765. https://doi.org/10.1016/j. actbio.2011.06.020

7. Arnesen S, Mosler S, Larsen NB et al (2009) The effects of collagen type I topography on myoblasts in vitro the effects of collagen type I topography on myoblasts in vitro:8207. https://doi.org/10.1080/ 03008200490888424

8. S. Ghanaati, M. Barbeck, A. Teiler, P. Booms, C. Kirkpatrick, R. Sader JL (2015) Collagen membranes induce different vascularization and cellular inflammatory response in relation to their origin in vivo and clinical studies. Int J Oral Maxillofac Surg 44:211. https://doi.org/10.1016/j.ijom.2015.08.088

9. Ghanaati S (2012) Non-cross-linked porcine-based collagen IIII membranes do not require high vascularization rates for their integration within the implantation bed: a paradigm shift. Acta Biomater 8:3061-3072. https://doi.org/10.1016/j.actbio. 2012.04.041

10. Ghanaati S, Kovács A, Barbeck M, Lorenz J, Teiler A, Sadeghi N, Kirkpatrick CJ, Sader R (2016) Bilayered, non-cross-linked collagen matrix for regeneration of facial defects after skin cancer removal: a new perspective for biomaterial-based tissue reconstruction. J Cell Commun Signal 10:3-15. https://doi.org/10.1007/ s12079-015-0313-7

11. Zhang Y, Al-Maawi S, Wang X et al (2018) Biomaterial-induced multinucleated giant cells express proinflammatory signaling molecules: a histological study in humans. J Biomed Mater Res A. https://doi.org/10.1002/jbm.a.36594

12. Al-Maawi S, Orlowska A, Sader R et al (2017) In vivo cellular reactions to different biomaterials - physiological and pathological aspects and their consequences. Semin Immunol 29:49-61. https:// doi.org/10.1016/J.SMIM.2017.06.001

13. Barbeck M, Lorenz J, Kubesch A et al (2015) Porcine dermis-derived collagen membranes induce implantation bed vascularization via multinucleated giant cells: a physiological reaction? J Oral Implantol 41: e238-e251. https://doi.org/10.1563/aaid-joi-D-14-00274

14. Plant AL, Bhadriraju K, Spurlin TA, Elliott JT (2009) Cell response to matrix mechanics: focus on collagen. Biochim Biophys Acta Mol Cell Res 1793:893-902. https://doi.org/10.1016/j.bbamcr. 2008.10.012

15. Schlee M, Ghanaati S, Willershausen I, Stimmlmayr M, Sculean A, Sader RA (2012) Bovine pericardium based non-cross linked collagen matrix for successful root coverage, a clinical study in human. Head Face Med 8:6. https://doi.org/10.1186/1746-160X-8-6

16. Rothamel D, Schwarz F, Sculean A et al (2004) Biocompatibility of various collagen membranes in cultures of human PDL fibroblasts and human osteoblast-like cells. Clin Oral Implants Res 15:443449. https://doi.org/10.1111/j.1600-0501.2004.01039.x

17. Chia-Lai PJ, Orlowska A, Al-Maawi S et al (2018) Sugar-based collagen membrane cross-linking increases barrier capacity of membranes. Clin Oral Investig 22:1851-1863. https://doi.org/10. 1007/s00784-017-2281-1

18. Barbeck M, Lorenz J, Holthaus MG, Raetscho N, Kubesch A, Booms P, Sader R, Kirkpatrick CJ, Ghanaati S (2015) Porcine dermis and pericardium-based, non-cross-linked materials induce multinucleated giant cells after their in vivo implantation: a physiological reaction? J Oral Implantol 41:e267-e281. https://doi.org/10. 1563/aaid-joi-D-14-00155

19. Duque GA, Descoteaux A (2014) Macrophage cytokines: involvement in immunity and infectious diseases. Front Immunol 5:1-12. https://doi.org/10.3389/fimmu.2014.00491

20. Mantovani A, Sica A, Sozzani S et al (2004) The chemokine system in diverse forms of macrophage activation and polarization:25. https://doi.org/10.1016/j.it.2004.09.015

21. Mills CD, Kincaid K, Alt JM et al (2017) Pillars article: M-1/M-2 macrophages and the Th1/Th2 Paradigm. J Immunol 199:2194 2201. https://doi.org/10.4049/jimmunol.1701141 
22. Barbeck M, Motta A, Migliaresi C et al (2016) Heterogeneity of biomaterial-induced multinucleated giant cells: possible importance for the regeneration process? J Biomed Mater Res A 104:413-418. https://doi.org/10.1002/jbm.a.35579

23. Ghanaati S, Orth C, Barbeck M et al (2010) Histological and histomorphometrical analysis of a silica matrix embedded nanocrystalline hydroxyapatite bone substitute using the subcutaneous implantation model in Wistar rats. Biomed Mater:5. https://doi.org/ 10.1088/1748-6041/5/3/035005

24. Wend S, Kubesch A, Orlowska A, al-Maawi S, Zender N, Dias A, Miron RJ, Sader R, Booms P, Kirkpatrick CJ, Choukroun J, Ghanaati S (2017) Reduction of the relative centrifugal force influences cell number and growth factor release within injectable PRFbased matrices. J Mater Sci Mater Med 28:28-11. https://doi.org/ 10.1007/s10856-017-5992-6

25. WHO (2010) WHO guidelines on drawing blood: best practices in phlebotomy. World Health Organ:1-105. https://doi.org/10.1038/ nature 16040

26. Miron R, Ghanaati S, Choukroun J (2018) Controversies related to scientific report describing $\mathrm{g}$-forces from studies on platelet-rich fibrin: necessity for standardization of relative centrifugal force values. Int J Growth Factors Stem Cells Dent 1:2-7. https://doi. org/10.4103/GFSC.GFSC

27. Kilkenny C, Browne WJ, Cuthill IC et al (2013) Improving bioscience research reporting: the arrive guidelines for reporting animal research. Animals 4:35-44. https://doi.org/10.3390/ani4010035

28. Brunel G, Piantoni P, Elharar F, Benqué E, Marin P, Zahedi S (1996) Regeneration of rat calvarial defects using a bioabsorbable membrane technique: influence of collagen cross-linking. J Periodontol 67:1342-1348. https://doi.org/10. 1902/jop.1996.67.12.1342

29. Al-Maawi S, Herrera-Vizcaíno C, Orlowska A et al (2019) Biologization of collagen-based biomaterials using liquid-plateletrich fibrin: new insights into clinically applicable tissue engineering. Materials (Basel) 12:3993. https://doi.org/10.3390/ ma12233993

30. Lorenz J, Al-maawi S, Sader R, Ghanaati S (2018) Individualized titanium mesh combined with platelet-rich fibrin and deproteinized bovine bone: a new approach for challenging augmentation individualized titanium mesh combined with platelet-rich fibrin and. $\mathrm{J}$ Oral Implantol. https://doi.org/10.1563/aaid-joi-D-18-00049

31. Ghanaati S, Herrera-Vizcaino C, Al-Maawi S et al (2018) Fifteen years of platelet rich fibrin in dentistry and oromaxillofacial surgery: how high is the level of scientific evidence? J Oral Implantol 44:471-492. https://doi.org/10. 1563/aaid-joi-D-17-00179

32. Albilia JB, Herrera-Vizcaíno C, Weisleder $\mathrm{H}$ et al (2018) Liquid platelet-rich fibrin injections as a treatment adjunct for painful temporomandibular joints: preliminary results. Cranio. https://doi.org/ 10.1080/08869634.2018.1516183

33. Al-Maawi S, Vorakulpipat C, Orlowska A et al (2018) In vivo implantation of a bovine-derived collagen membrane leads to changes in the physiological cellular pattern of wound healing by the induction of multinucleated giant cells: an adverse reaction? Front Bioeng Biotechnol 6:1-13. https://doi.org/10. 3389/fbioe. 2018.00104

34. Herrera-Vizcaíno C, Dohle E, Al-Maawi S et al (2019) Platelet-rich fibrin secretome induces three dimensional angiogenic activation in vitro. Eur Cells Mater 37. https://doi.org/10.22203/eCM. v037a15

35. Kubesch A, Barbeck M, Al-Maawi S et al (2018) A low-speed centrifugation concept leads to cell accumulation and vascularization of solid platelet-rich fibrin: an experimental study in vivo. Platelets 00:1-12. https://doi.org/10.1080/09537104.2018. 1445835
36. Verboket R, Herrera-Vizcaíno C, Thorwart K et al (2018) Influence of concentration and preparation of platelet rich fibrin on human bone marrow mononuclear cells (in vitro). Platelets. https://doi.org/ 10.1080/09537104.2018.1530346

37. Zhang D, Wu X, Chen J, Lin K (2018) The development of collagen based composite scaffolds for bone regeneration. Bioact Mater 3:129-138. https://doi.org/10.1016/j.bioactmat.2017.08.004

38. Golomb G (1992) Calcification of polyurethane-based biomaterials implanted subcutaneously in rats: role of porosity and fluid absorption in the mechanism of mineralization. J Mater Sci Mater Med 3: 272-277. https://doi.org/10.1007/BF00705292

39. Gallagher WM, Lynch I, Allen LT, Miller I, Penney SC, O'Connor DP, Pennington S, Keenan AK, Dawson KA (2006) Molecular basis of cell-biomaterial interaction: insights gained from transcriptomic and proteomic studies. Biomaterials 27:58715882. https://doi.org/10.1016/j.biomaterials.2006.07.040

40. Barbero F, Russo L, Vitali M, Piella J, Salvo I, Borrajo ML, Busquets-Fité M, Grandori R, Bastús NG, Casals E, Puntes V (2017) Seminars in immunology formation of the protein corona: the Interface between nanoparticles and the immune system. Semin Immunol 34:52-60. https://doi.org/10.1016/j.smim.2017.10.001

41. Sadtler K, Wolf MT, Ganguly S et al (2018) Divergent immune responses to synthetic and biological scaffolds. Biomaterials. $\mathrm{https} / / /$ doi.org/10.1016/J.BIOMATERIALS.2018.11.002

42. Ferland-McCollough D, Slater S, Richard J, Reni C, Mangialardi G (2017) Pericytes, an overlooked player in vascular pathobiology. Pharmacol Ther 171:30-42. https://doi.org/10.1016/j.pharmthera. 2016.11.008

43. Martin D, Galisteo R, Gutkind JS (2009) CXCL8/IL8 stimulates vascular endothelial growth factor (VEGF) expression and the autocrine activation of VEGFR2 in endothelial cells by activating NFKB through the CBM (Carma3/Bcl10/Malt1) complex. J Biol Chem 284:6038-6042. https://doi.org/10.1074/jbc.C800207200

44. Sheikh Z, Brooks PJ, Barzilay O, Fine N, Glogauer M (2015) Macrophages, foreign body giant cells and their response to implantable biomaterials. Materials (Basel) 8:5671-5701. https://doi. org/10.3390/ma8095269

45. Janmey PA, McCulloch CA (2007) Cell mechanics: integrating cell responses to mechanical stimuli. Annu Rev Biomed Eng 9:1-34. https://doi.org/10.1146/annurev.bioeng.9.060906.151927

46. Ghanaati S, Barbeck M, Orth C, Willershausen I, Thimm BW, Hoffmann C, Rasic A, Sader RA, Unger RE, Peters F, Kirkpatrick CJ (2010) Influence of $\beta$-tricalcium phosphate granule size and morphology on tissue reaction in vivo. Acta Biomater 6:44764487. https://doi.org/10.1016/j.actbio.2010.07.006

47. Ghanaati S, Kirkpatrick CJ, Kubesch A et al (2014) Induction of multinucleated giant cells in response to small sized bovine bone substitute (Bio-Oss ${ }^{\mathrm{TM}}$ ) results in an enhanced early implantation bed vascularization. Ann Maxillofac Surg 4:150-157. https://doi. org/10.4103/2231-0746.147106

48. Cliche S, Amiot J, Avezard C, Gariépy C (2003) Extraction and characterization of collagen with or without telopeptides from chicken skin. Poult Sci 82:503-509. https://doi.org/10.1093/ps/82.3.503

49. Preethi Soundarya S, Haritha Menon A, Viji Chandran S, Selvamurugan N (2018) Bone tissue engineering: scaffold preparation using chitosan and other biomaterials with different design and fabrication techniques. Int J Biol Macromol 119:1228-1239. https://doi.org/10.1016/j.ijbiomac.2018.08.056

50. Bukoreshtliev NV, Haase K, Pelling AE (2013) Mechanical cues in cellular signalling and communication. Cell Tissue Res 352:77-94. https://doi.org/10.1007/s00441-012-1531-4

Publisher's note Springer Nature remains neutral with regard to jurisdictional claims in published maps and institutional affiliations. 